\title{
Sources of linear and non-linear synchrony between brain and muscles
}

\author{
Linear and non-linear CMC sources
}

\author{
Carmen Vidaurre \\ Dept. of Statistics, Informatics and Mathematics, Public \\ University of Navarre, Pamplona, Spain \\ Machine Learning Dept. TU-Berlin, Berlin, Germany \\ Guido Nolte \\ Dept. of Neurophysiology and Pathophysiology, \\ University Medical Center Hamburg-Eppendorf, \\ Hamburg, Germany \\ Katherina von Carlowitz-Ghori \\ Dept. of Neurology, Charité University Hospital, Berlin \\ Germany
}

\author{
Marisol Gómez \\ Dept. of Statistics, Informatics and Mathematics, Public \\ University of Navarre, Pamplona, Spain \\ Arno Villringer \\ Dept. of Neurology, Max Planck Institute for Human \\ Cognitive and Brain Sciences, Leipzig, Germany \\ Vadim V. Nikulin \\ Dept. of Neurology, Max Planck Institute for Human \\ Cognitive and Brain Sciences, Leipzig, Germany \\ Neuroscience, National Research University Higher \\ School of Economics, Moscow, Russian Federation
}

\begin{abstract}
This manuscript shows that it is possible to find distinct sources of brain activity, at similar frequencies, arising from linear and non-linear interactions of the brain with the muscular system. Those sources were obtained by maximizing coherence between multivariate signals recorded from brain and a single channel from the muscles. To find linear phase synchrony we used unrectified electromyographic recordings, whereas to de-mix nonlinear sources, we used rectified muscular measurements. In order to obtain the brain sources, we employed a recently published method called "cacoh" that is able to maximize coherence over the complete frequency range of interest and simultaneously find patterns of sources for each them. Our results show that cortico-muscular interactions even at the same frequency range can have spatially distinct neuronal sources depending on whether interactions had linear or non-linear character.
\end{abstract}

Keywords- linear interactions, non-linear interactions, corticomuscular coherence; brain activity; muscular activity;

\section{INTRODUCTION}

Over approximately the last 25 years, research has extensively shown that synchrony between oscillatory signals is related to effective communication between neuronal populations and is associated with large-scale integration [1-4]. Large-scale synchronization can also be found between cortical and muscular signals and previous studies showed that it is an effective way to estimate the communication between cortex and spinal cord [5-7]. Oscillations in the beta frequency range (between 15 and $30 \mathrm{~Hz}$ ) have been associated with different aspects of motor control. In this respect, cortico-muscular coherence (CMC) offers the possibility to non-invasively study the coupling between sensorimotor cortices and muscle activity
[8-10]. CMC estimates the phase coupling between cortical and muscular activities at specific frequency bands. It reaches its maximum during the isometric intervals of the movement, whereas it decreases for the kinematic parts [11,12]. CMC has been assessed in healthy population using magnetoencephalography (MEG) $[13,14]$ as well as electroencephalography (EEG) [15-19]. CMC detection remains challenging because of its weak strength as well as distributed cortical origin. In order to improve CMC estimation, we recently published a new method called canonical coherence (caCOH) [20]. caCOH directly maximizes the absolute value of the coherence between two multivariate spaces of different origin in the frequency domain. This allows very fast optimization for multiple frequency bins. Furthermore, caCOH directly finds topographies of only those neuronal sources that are coupled to maximal coherence. Thus, the time courses of $\mathrm{caCOH}$ components directly show activity of neuronal sources. In order to recover the exact spatial location of these sources in the brain source modelling using the obtained $\mathrm{caCOH}$ patterns can be used. caCOH topographies are different from the usual patterns of coherence that only offer information about the distribution of coherence on the scalp, but not about its neural sources. Moreover, currently there is a debate about the rectification of electromyogram (EMG) for the calculation of corticomuscular coherence (CMC), [21]. Yet there are no studies showing whether EMG rectification may lead to neuronal sources being associated with different neuronal populations. Taking advantage of the aforementioned, in this work, we apply caCOH to study CMC on the basis of un-rectified (linear interactions) or rectified (non-linear) muscle activity has distinct cortical spatial sources at the same frequency bin If these sources are different, it would indicate that separate neuronal processes are

C.V gratefully acknowledges funding from MINECO, RYC-2014-15671, G.N. was partially sponsored by DFG, SFB936 Z3 and TRR169, B4. V.V.N funding is from HSE Basic Research Program, Russian Academic Excellence Project '5-100'. 
responsible for the generation of linear and non-linear $\mathrm{CMC}$ interactions.

\section{METHODS}

\section{A. Maximizatin of Canonical Coherence}

caCOH maximizes the absolute value of coherence between two multivariate spaces $A$ and $B$ of dimensions $N_{A}$ and $N_{B}$ respectively. The goal is to find a real valued linear combination of sensors of set A and a real valued linear combination of sensors of set B such that the absolute value of coherence between the two virtual sensors is maximized at a specific frequency. After the mathematical developments presented in [20], it can be shown that for each phase, the optimization problem boils down to a generalized eigenvalue decomposition. However, it is necessary to estimate the phase of maximum coherence. For that, a non-linear numerical search is performed, that allows us arrive to an essentially exact solution after a low number of iterations (please refer to [20] for more details). As previously mentioned, the optimization procedure also returns the corresponding topographies from the linear filters that optimize coherence.

In order to locate the cortical sources corresponding to the patterns of sources obtained with $\mathrm{caCOH}$ we applied inverse modeling with eLORETA [22].

Finally, we computed the difference between two different patterns $\left(\mathrm{a}_{1}\right.$ and $\left.\mathrm{a}_{2}\right)$ according to:

$$
\text { Diff }=1-\frac{\left|a_{1}^{T} a_{2}\right|}{\left\|a_{1}\right\|\left\|a_{2}\right\|}
$$

\section{B. Dataset}

Data were recorded from 14 healthy volunteers without history of neurological or psychiatric disorders. The experimental protocol was approved by the Institutional Review Board of Charité, Berlin, and participants gave their written informed consent prior to the recordings. All subjects were righthanded according to the Edinburg Handedness Inventory [23] and had normal or corrected to normal vision. These data were already described in [7].

\section{Paradigm}

The participants performed a digit displacement paradigm, that has been reported to increase CMC values in comparison to other paradigms not involving a compliant lever $[11,12]$. The participants had to move the aforementioned lever against a load and then maintain a constant force (digit displacement). Volunteers were seated in a comfortable chair with their arms rested on the chair handles, forearms flexed at $60^{\circ}$ and hands pronated. Participants had to press a spring-loaded lever with the left or right thumb at $0.5 \mathrm{~N}$ force. The force level was measured with a Honeywell Load Sensor (FSG15N1A) and visually presented to the participants as a horizontal bar proportional to the exerted force. A cross in the center of the screen was also displayed and served as eye-fixation point. The displacement of the spring-loaded lever was $3.5 \mathrm{~cm}$. The volunteers had to perform the task with each hand separately and the order was counter-balanced between participants. The volunteers were instructed to reach the desired force level as fast as possible after a single tone was presented and hold the force constant until a double tone was presented. One hundred trials were recorded for each hand in four blocks. Each trial lasted $9 \mathrm{~s}$ in total $(5 \mathrm{~s}$ active and $4 \mathrm{~s}$ rest). There were $60 \mathrm{~s}$ of rest between the blocks.

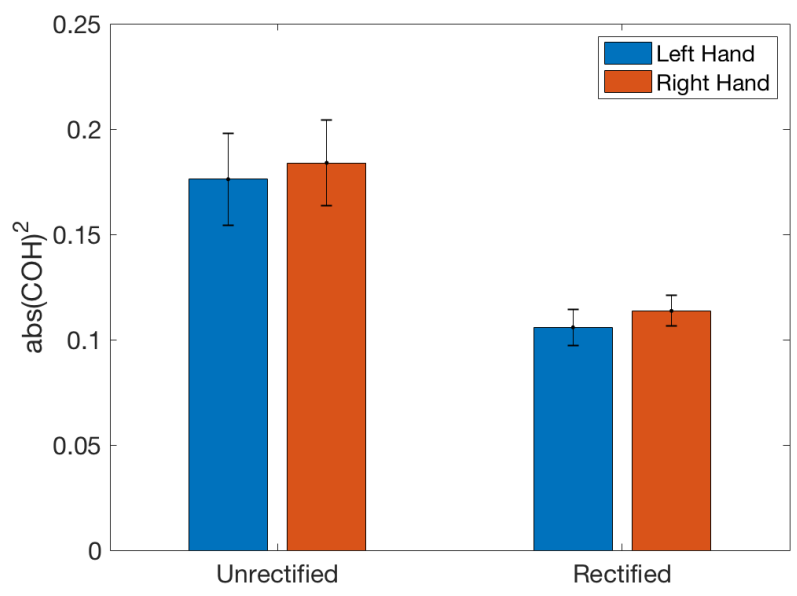

Figure 1. Bar plot of averaged maximal $\mathrm{CMC}$ values obtained with $\mathrm{caCOH}$ for rectified and unrectified EMG. Results for left and right hand are depicted separately

\section{Data acquisition and preprocessing}

A BrainAmp MR plus from Brain Products (Germany) was used to record EEG and EMG data. The signals were filtered between 0.015 and $250 \mathrm{~Hz}$ with a voltage resolution of $0.1 \mu \mathrm{V}$ for EEG and $0.5 \mu \mathrm{V}$ for EMG. The sampling frequency as 1000 $\mathrm{Hz}$ but the data was later band pass filtered and down-sampled to $200 \mathrm{~Hz}$. The EEG data was referenced to physically linked earlobes. An EEG cap (EasyCapTM) with $61 \mathrm{Ag} / \mathrm{AgCl}$ sintered ring electrodes (12 mm diameter, EasyCap GmbH, Germany) densely covering the sensorimotor cortex was employed to record EEG signals. EOG signals were also acquired with two electrodes placed on the right zygomatic and supraorbital processes in order to assess ocular artifacts. EMG signals were acquired with 6 EMG electrodes placed over the thenar side of each hand and from the Abductor Pollicis Brevis (APB) muscle. These electrodes were $\mathrm{Ag} / \mathrm{AgCl}$ sintered sensors with $4 \mathrm{~mm}$ diameter. The EMG reference electrode was placed on the styloid process of the ulnar bone and a ground electrode on the inner surface of wrist at the midline, with $\mathrm{Ag} / \mathrm{AgCl}$ sintered sensors of $12 \mathrm{~mm}$ diameter. The data analysis was performed in MATLAB (2018a; The MathWorks, Natick, MA) using the BBCI toolbox [24] the Fieldtrip toolbox [25], the EEGlab toolbox [26] and custom programmed software.

CMC was estimated during the post-stimulus interval between 2 and $5 \mathrm{~s}$, which corresponded to the stable hold period of the task. This is the interval at which the strongest coherent activity in beta band can be expected [5, 12, 27]. Before CMC calculation, artefactual trials were rejected (see [7]). CMC was computed by means of $\mathrm{caCOH}$ in two different ways: 1) using the raw EMG signal of the best electrode as selected in [7]. 2) using the rectified EMG signal of the same electrode as in 1). Rectification was achieved by high pass filtering the sensor data above $10 \mathrm{~Hz}$ and then computing the absolute value of the resulting high-pass filtered signal. 

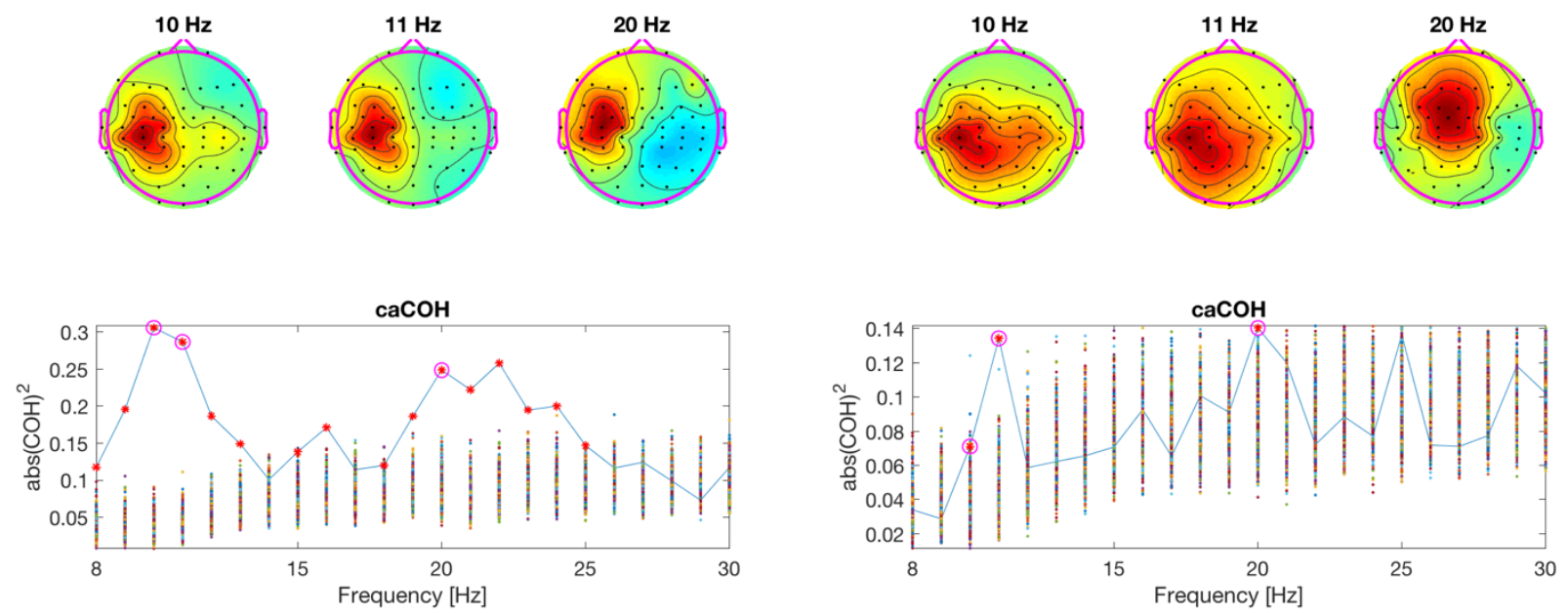

Figure 2. Up: selected patterns of sources for three frequency bins. The maximized CMC values at those bins are significantly different from random for both unrectified (left) and rectified (right) EMG signals. Bottom: spectrum of maximized $\mathrm{CMC}$ with caCOH. All values marked with red stars are significantly different from random. Values marked with magenta circles are those selected to display the corresponding patterns of sources. Vertical lines corespond to $500 \mathrm{CMC}$ values from computing $\mathrm{caCOH}$ with shuffled data 500 times.

\section{E. Significance estimation}

We assessed the significance of coherence with permutation tests [28] This procedure consisted of repeating all steps of caCOH, but the EMG segments were shuffled with respect to the EEG data. This was repeated 500 times. Significant $C M C$ values of the original data were those exceeding the 97.5 percentile of permuted coherence.

\section{RESULTS}

\section{A. caCOH maximal values with rectified and unrectified $E M G$}

We performed the maximization of coherence between EEG and one EMG channel, where the EMG signal was either raw or rectified. In the case of raw EMG, the results were significantly different from random for 24 datasets (thus, results were not significant in 4 occasions). For the rectified EMG, CMC was significantly different from random for 12 datasets (and 16 times the result was not significant). Using $\mathrm{caCOH}$, the average maximal peak obtained with the best raw EMG channel was $0.18 \pm 0.02$ for both left and right hand. In the case of rectified EMG, the average maximum coherence was $0.11 \pm 0.01$ for both left and right hands. When the EMG was rectified, the coherence was significantly lower for both hands in comparison to unrectified EMG (p-values $<0.001$ in both cases using twotailed Wilcoxon tests).

The grand-average over subject of maximal values obtained with caCOH for both analyses are visible in Figure 1.

\section{B. caCOH with unrectified and rectified EMG}

We selected one of the subjects with significant results for the right hand in the rectified case to exemplify source differences estimated from linear and non-linear interactions. In Figure 2 we plot the spectrum of maximized coherence obtained for caCOH (bottom). The values on the spectrum marked with a red star are those significantly different from random at $97.5 \%$ of confidence. The values circled in magenta were selected to display their corresponding patterns. The corresponding sources of patterns maximizing coherence at the frequency bins marked with magenta circles are depicted in the upper part of the plot. The corresponding located sources of the patterns depicted in Fig. 2 are visible in Figure 3. On the left side, we plotted the sources obtained with unrectified EMG. The sources obtained with rectified EMG are on the right of the picture. Each frequency bin is displayed in a different row. While we see some similarities between the patterns corresponding to rectified and un-rectified EMG (predominantly contralateral location of the sources) we also see some differences such as more widespread and even bilateral presence of neuronal sources in case of unrectified EMG. A visual inspection of sources from other subjects further confirmed a presence of dissimilarities between the $\mathrm{CMC}$ for rectified and unrectified EMG.

\section{DISCUSSION}

The previous results show that there exist significant differences in maximizing the coherence between brain and muscles using unrectified or rectified EMG data. The number of significant CMS was lower and the number of frequency bins for which CMC is different from random is fewer for the rectified case. Importantly in this study we confirmed it for a broad frequency range, not being limited to the beta frequencies. Furthermore, as we see in Figure 3, the location of coherent sources is also different, even for the same frequency bins. This result is especially obvious for the sources at $20 \mathrm{~Hz}$, in the beta frequency band, for which the difference between both patterns was 0.56 (with the maximum difference being 1 and the minimum 1). Thus, from these results we suggest that the sources of linear and non-linear interaction differ, and they are likely to relate to different brain processes. Unrectified EMG more directly relates to the generated strength and due to this we 
can also see activity in the ipsilateral motor cortex. This is most likely due to a possibility of generation of physiological mirror activity [29]. Future studies can explore this aspect in more details using stronger forces which are known to be associated with larger physiological mirror activity. In this case, we would expect to see even stronger activity in the ipsilateral sensorimotor cortex.
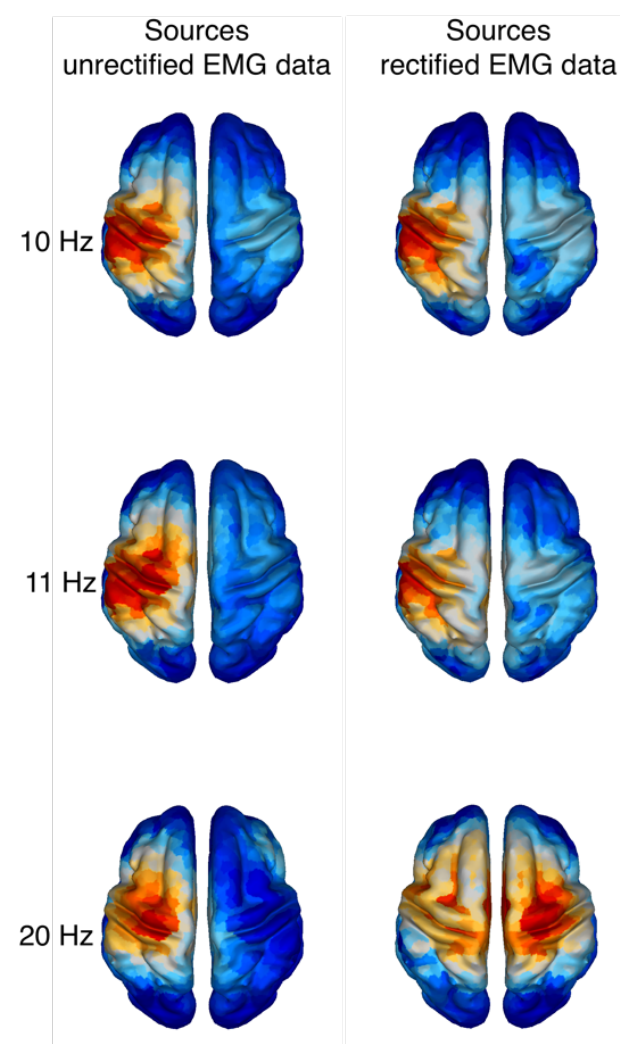

Figure 3. Sources obtained using unrectified (left) and rectified (right) EMG data. Sources were located using eLORETA. Each selected frequency bin corresponds to one row.

\section{ACKNOWLEDGMENT}

The authors gratefully acknowledge Klaus-Robert Müller for fruitful discussions. We also thank all co-authors of reference [20] for allowing re-usage of some text fragments in that publication.

\section{REFERENCES}

[1] P. Fries, "A mechanism for cognitive dynamics: neuronal communication through neuronal coherence.” Trends Cogn Sci. vol. 9, num. 10, pp. 474480, 2005.

[2] A. K. Engel, P. R. Roelfsema, P. Fries, M. Brecht, W. Singer, "Role of the temporal domain for response selection and perceptual binding." Cerebral Cortex, vol. 7 num. 6, pp. 571-582, 1997.
[3] F. Varela, J. P. Lachaux, E. Rodriguez, J. Martinerie. "The brainweb: phase synchronization and large-scale integration." Nat Rev Neurosci vol. 2 num. 4, pp. 229-239, 2001.

[4] V.V. Nikulin, K. Linkenkaer-Hansen, G. Nolte, S. Lemm, K.-R- Müller, R.J. Ilmoniemi, G. Curio, "A novel mechanism for evoked responses in the human brain.” European Journal of Neuroscience, vol. 25, num. 10, pp. 3146-3154, 2007

[5] S. N. Baker, E. Olivier, R. N. Lemon, R. N., "Coherent oscillations in monkey motor cortex and hand muscle emg show task-dependent modulation.” J. Physiol., vol.501 num. 1, pp. 225-241, 1997.

[6] J. M. Schoffelen, R. Oostenveld, P. Fries, "Imaging the human motor system's beta-band synchronization during isometric contraction." Neuroimage vol. 41 num. 2, pp. 437-447, 2008.

[7] Z. Bayraktaroglu, K. von Carlowitz-Ghori, F. Losch, G. Nolte, G. Curio, V. V. Nikulin, V. V.,. "Optimal imaging of cortico-muscular coherence through a novel regression technique based on multi-channel eeg and unrectified emg." Neuroimage vol. 57, pp. 1059-1067, 2011

[8] S. N. Baker, "Oscillatory interactions between sensorimotor cortex and the periphery." Curr. Opin. Neurobiol vol. 17 num. 6, pp. 649-655, 2007.

[9] A. Jackson, R. L. Spinks, T. C. B. Freeman, D. M. Wolpert, R. N. Lemon, "Rhythm generation in monkey motor cortex explored using pyramidal tract stimulation.” J. Physiol. vol. 541 num. 3, pp. 685-699, 2002.

[10] A. Schnitzler, J. Gross, L. Timmermann,." Synchronised oscillations of the human sensorimotor cortex." Acta Neurobiol vol. 60 num. 2, pp. 271 $287,2000$.

[11] J. M. Kilner, S. N. Baker, S. Salenius, R. Hari, R. N. Lemon. "Human cortical muscle coherence is directly related to specific motor parameters." J. Neurosci vol. 20 num. 23, pp. 8836-8845, 2000.

[12] C. N. Riddle, S. N. Baker, "Manipulation of peripheral neural feed- back loops alters human corticomuscular coherence." J. Physiol vol. 566 num. 2, pp. 625-639, 2005.

[13] B. A. Conway, D. M. Halliday, S. F. Farmer, U. Shahani, P. Maas, A. I. Weir, J. R. Rosenberg, "Synchronization between motor cortex and spinal motoneuronal pool during the performance of a maintained motor task in man." J. Physiol, vol. 489 num. 3, pp. 917-924, 1995.

[14] S. Salenius, K. Portin, M. Kajola, R. Salmelin, R. Hari, "Cortical control of human motoneuron firing during isometric contraction." J. Neurophysiol vol. 77 num. 6, pp. 3401-3405, 1997.

[15] M. R. Baker, S. N. Baker, "The effect of diazepam on motor cortical oscillations and corticomuscular coherence studied in man.” J. Physiol., vol. 546 num. 3, pp. 931-942, 2002.

[16] S. Graziadio, A. Basu, L. Tomasevic, F. Zappasodi, F. Tecchio, J. A. Eyre, "Developmental tuning and decay in senescence of oscillations linking the corticospinal system." J. Neurosci., vol. 30 num. 10, pp. 3663-3674, 2010.

[17] D. M. Halliday, B. A. Conway, S. F. Farmer, J. R. Rosenberg. "Using electroencephalography to study functional coupling between cortical activity and electromyograms during voluntary contractions in humans." Neurosci. Lett. vol. 241, num. 1, pp. 5-8, 1998.

[18] R. Kristeva-Feige, C. Fritsch, J. Timmer, C.-H. Lücking, C.-H, "Effects of attention and precision of exerted force on beta range eeg-emg synchronization during a maintained motor contraction task." Clin. Neurophysiol vo. 113 num. 1, pp. 124-131, 2002.

[19] T. Mima, M. Hallett, "Electroencephalographic analysis of corticomuscular coherence: reference effect, volume conduction and generator mechanism." Clin. Neurophysiol vol. 110 num. 11, 1892-1899, 1999.

[20] C. Vidaurre, G. Nolte, I.E.J. de Vries, M. Gómez, T. W. Boonstra, K-R. Müller, A. Villringer, V. V. Nikulin "Canonical maximization of coherence: A novel tool for investigation of neuronal interactions between two datasets." Neuroimage, vol. 201, pp. 116009, 2019.

[21] V. M. McClelland, Z. Cvetkovic, K. R. Mills, "Rectification of the EMG is an unnecessary and inappropriate step in the calculation of Corticomuscular coherence.” J Neurosci Methods, vol. 205, pp.190-201, 2012.

[22] R. D. Pascual-Marqui, D. Lehmann, M. Koukkou, K. Kochi, P. Anderer, B. Saletu, H. Tanaka, K. Hirata, E. John, E., L. Prichep, R. Biscay-Lirio, T. Kinoshita, "Assessing interactions in the brain with exact low- 
resolution electromagnetic tomography." Philos Trans A Math Phys Eng Sci vol. 369, num. 1952, pp. 3768-3784, 2011.

[23] R. C. Oldfield, R. C, "The assessment and analysis of handedness: the edin- burgh inventory." Neuropsychologia vol. 9 num. 1, pp. 97-113, 1971.

[24] B. Blankertz, L. Acqualagna, S. Dähne, S. Haufe, M. Schultze-Kraft, I. Sturm, M. Uscumlic, M. Wenzel, G. Curio, K.-R., Müller, "The berlin brain-computer interface: Progress beyond communication and control." Frontiers in Neuroscience, vol. 10, 2016.

[25] R. Oostenveld, P. Fries, E. Maris, J.-M. Schoffelen, J.-M, "Fieldtrip: Open source software for advanced analysis of MEG, EEG, and invasive electrophysiological data." Computational Intelligence and Neuroscience, 156869,2011

[26] A. Delorme, S. Makeig, "EEGLAB: an open source toolbox for analysis of single-trial eeg dynamics." Journal of Neuroscience Methods, vol. 134, pp. 9-21, 2004
[27] R. Kristeva, L. Patino, W. Omlor, "Beta-range cortical motor spectral power and corticomuscular coherence as a mechanism for effective corticospinal interaction during steady-state motor output." Neuroimage vol. 36 num. 3, pp. 785-792, 2007.

[28] T. Hesterberg, D. Moore, S. Monaghan, A. Clipson, R. Epstein. Bootstrap methods and permutation tests. In: Moore, D., McCabe, G. (Eds.), Introduction to the Practice of Statistics. W.H. Freeman, New York, pp. 14.1-14.70, 2005.

[29] T. Maudrich, R. Kenville, V. V. Nikulin, D. Maudrich, A. Villringer, P. Ragert, "Inverse relationship between amplitude and latency of physiological mirror activity during repetitive isometric contractions." Neuroscience, vol. 406, pp. 300-313, 2019. 